\title{
Novedades para la orquidoflora de la provincia de Segovia.
}

\section{New data cocerning orchid flora in the province of Segovia.}

Teófilo Martín-Gil ${ }^{1}$, Jaime Gila-Marazuela ${ }^{2}$

\section{Resumen}

A partir de los resultados obtenidos de la extensa e intensa prospección del territorio, se presenta información corológica sobre 9 taxones de la familia Orchidaceae en la provincia de Segovia, Coeloglossum viride (L.) Hartm., Epipactis helleborine (L.) Crantz subsp. helleborine, Epipactis microphylla (Ehrh.) Swartz., Epipactis tremolsii Pau, Ophrys fusca Link. subsp. fusca, Ophrys insectifera L. subsp. insectifera, Orchis papilionacea L., Orchis ustulata L. y Spiranthes spiralis (L.) Chevall, así como del híbrido Orchis x gennarii Reichenb. fil. (O. morio x O. papilionacea), los cuales además de constituir primeras citas provinciales, amplían notablemente el conocimiento de la orquidoflora silvestre de Segovia. Se aportan datos sobre su distribución en la CCAA de Castilla y León, ecología, estado de las poblaciones y conservación. Así mismo, se incluyen mapas de distribución en cuadrículas UTM de $10 \times 10 \mathrm{Km}$ y material fotográfico.

Palabras clave: Orchidaceae, corología, Segovia, CCAA Castilla y León, España.

\section{Abstract}

Based on the results obtained from intense and extensive prospecting of the territory chorological information is hereafter put forward for 9 taxa from the Orchidaceae family in the province of Segovia, Coeloglossum viride (L.) Hartm., Epipactis helleborine (L.) Crantz

\footnotetext{
1 Agente Medioambiental y Celador de Medio Ambiente. C/Miguel de Unamuno $n^{\circ} 7$, Bloque I, $1^{\circ}$ A, Boadilla del Monte, 28660, Madrid (España).

*Correspondencia: teomartingil@telefonica.net

2 Profesor. C.E.I.P. Marqués del Arco. C/ Salineros n²7, Mozoncillo, 40250, Segovia (España).

*Correspondencia: jjjaimesss@hotmail.com
} 
subsp. helleborine, Epipactis microphylla (Ehrh.) Swartz., Epipactis tremolsii Pau, Ophrys fusca Link. subsp. fusca, Ophrys insectifera L. subsp. insectifera, Orchis papilionacea L., Orchis ustulata L. and Spiranthes spiralis (L.) Chevall, as well as for the hybrid Orchis $x$ gennarii Reichenb. fil. (O. morio x O. papilionacea), which, besides representing the first populations found in the province, noticeably increase the knowledge about the wild orchid flora in the province of Segovia. Data concerning their distribution, ecology, state of the populations and conservation in the Castilla y León region is brought forward. Also included is photographic material and distribution maps in 10x10 Km UTM grid cells.

Key words: Orchidaceae, chorology, Segovia, CCAA Castille y Leon, Spain.

\section{Laburpena}

Hango lurraldean egindako azterketa zabal eta sakonaren ondoren, Segoviako Orchidaceae familiako 9 taxoiren informazio korologikoa aurkezten da lan honetan. Coeloglossum viride (L.) Hartm., Epipactis helleborine (L.) Crantz subsp. helleborine, Epipactis microphylla (Ehrh.) Swartz., Epipactis tremolsii Pau, Ophrys fusca Link. subsp. fusca, Ophrys insectifera L. subsp. insectifera, Orchis papilionacea L., Orchis ustulata L. y Spiranthes spiralis (L.) Chevall, eta bai Orchis x gennarii Reichenb. fil. (O. morio x O. papilionacea) hibridoarena ere. Probintzian lehen zitak izateaz gain, Segoviako basa orkidofloraren ezagutza zabaltzeko urrats handia dira. Gaztela eta Leongo Autonomia Erkidegoan espezieek duten banaketaz, ekologiaz, populazioen egoeraz eta kontserbazioaz ere datuak aurkezten dira lan honetan, baita 10 × 10 Km UTM kuadrikuletan banaketa mapak eta argazki-materiala ere.

Gako hitzak: Orchidaceae, korologia, Segovia, Gaztela eta Leongo Autonomia Erkidegoa, Espainia.

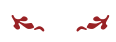

El contexto geográfico de la provincia de Segovia (Sector Centro Norte del Sistema Central), se encuentra todavía poco prospectado desde el punto de vista del estudio y conocimiento de su flora vascular, careciendo de un catálogo florístico completo y actualizado. Tan sólo existen algunos trabajos parciales destacables que abarcan áreas geográficas restringidas (Cebolla et al. 1984; Romero \& Rico, 1989; García Adá, 1995; Gabriel y Galán \& Puelles, 1996; Soriano et al. 2002) y diversas notas florísticas, no existiendo trabajos específicos sobre la familia Orchidaceae. En las últimas décadas ha aumentado considerablemente el interés de botánicos, naturalistas y aficionados por el estudio y conocimiento de las orquídeas silvestres. Esta corriente de interés se ha traducido en la publicación de numerosos estudios y trabajos en diversidad de territorios, tanto a escala regional como provincial e incluso local. Prueba de ello, son los trabajos realizados y publicados en nuestro entorno más inmediato, Valladolid 
(Santos et al. 2008), Zamora (Bariego et al. 2005), y Palencia (Ruiz de Gopegui et al. 2017), que han contribuido de manera notable a ampliar y profundizar en el conocimiento corológico, ecológico y demográfico de las distintas especies de orquídeas silvestres presentes, aspectos todos ellos clave de cara a la gestión y conservación de sus poblaciones.

A raíz de los exhaustivos trabajos de prospección botánica que se vienen desarrollando en el ámbito de la provincia de Segovia, dirigidos a profundizar en el conocimiento sobre la distribución de los distintos taxones de la familia Orchidaceae, con el propósito de elaborar un catálogo de las orquídeas silvestres de la provincia, se ha incrementado considerablemente el conocimiento sobre la orquidoflora segoviana. En este sentido, y como adelanto del mismo, se presenta información corológica de 9 taxones, Coeloglossum viride (L.) Hartm., Epipactis helleborine (L.) Crantz subsp. helleborine, Epipactis microphylla (Ehrh.) Swartz., Epipactis tremolsii Pau, Ophrys fusca Link. subsp. fusca, Ophrys insectifera L. subsp. insectifera, Orchis papilionacea L., Orchis ustulata L. y Spiranthes spiralis (L.) Chevall, que no han sido citados hasta ahora en la provincia, con tan sólo una cita muy antigua (C. viride) o reciente no confirmada posteriormente (O. papilionaceea), o bien en algunos casos que han sido citados erróneamente (E. tremolsii). Además se aportan las primeras citas del híbrido Orchis x gennarii Reichenb. fil. (O. morio x O. papilionacea). Varias de las orquídeas silvestres citadas están consideradas raras y escasas en la Comunidad castellano-leonesa, siendo esta circunstancia reconocida mediante su catalogación e inclusión en listados de flora protegida y la asignación de un régimen singular de protección (Decreto 63/2007; Ley 4/2015).

Respecto al tratamiento taxonómico, nomenclatura y autoría se ha adoptado el criterio de Flora Iberica Vol. XXI (Aedo \& Herrero, 2005). En cuanto a la corología, se ha utilizado con carácter general Flora Iberica (Aedo \& Herrero, 2005) y la base de datos de ANTHOS (Anthos, 2018), mientras que para el ámbito geográfico castellano-leonés se han tomado como referencia de consulta las Bases de Datos del Catálogo de Flora vascular Silvestre de Castilla y León (http://jcyl1.pluntic.es/index.php/bbdd-floravascular-silvestre, Junta de Castilla y León, 2001-2013); los principales trabajos botánicos en el marco provincial (Cebolla et al. 1984; Romero \& Rico, 1989; García Adá, 1995; Gabriel y Galán \& Puelles, 1996; Soriano et al. 2002); los catálogos y listados florísticos de las provincias limítrofes, Burgos (Alejandre et al. 2006), Guadalajara (Carrasco et al. 1997) y Soria (Segura et al. 2000); y varios trabajos monográficos sobre la familia Orchidaceae realizados en el entorno geográfico más próximo (Bariego \& Gastón, 2005; Santos et al. 2008; Ruiz de Gopegui et al. 2017).

De cada uno de los taxones, ordenados alfabéticamente, se indica nombre científico - autoría; información relativa a la localización en coordenadas UTM (datum ETRS89), en cuadrícula de 1×1 Km ó bien de 10×10 Km cuando procede un tratamiento discreto de información sensible por tratarse de taxones raros y/o con poblaciones muy escasas; municipio, localidad y topónimo; observaciones sobre el hábitat, altitud, fecha, autores 
de la observación y referencia de la fotografía testigo que respalda la cita (Fig. 1). Tal y como se ha señalado, en ningún caso se herborizó material, decisión adoptada por dos poderosas razones. En primer lugar, debido a que se citan especies de orquídeas raras y con poblaciones muy exiguas, que en algunos casos están constituidas por un reducido número de ejemplares (1-7). Y en segundo lugar, porque el material de los ejemplares prensados no mantiene en buen estado los caracteres diagnósticos de la especie a la que corresponde, siendo estos perfectamente reconocibles con una fotografía realizada en plena floración mediante técnicas de macrofotografía.

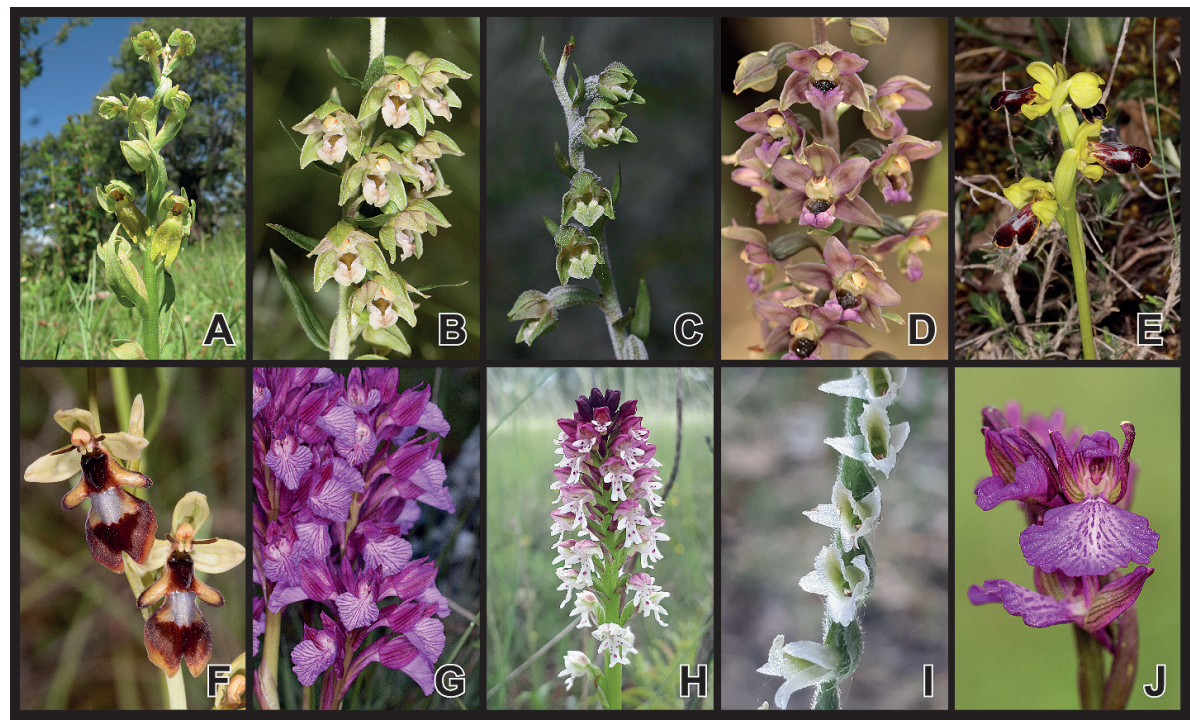

Fig. 1.- A. Coeloglossum viride (L.) Hartm.; B. Epipactis helleborine (L.) Crantz subsp. helleborine; C. Epipactis microphylla (Ehrh.) Swartz.; D. Epipactis tremolsii Pau; E. Ophrys fusca Link. subsp. fusca; F. Ophrys insectifera L. subsp. insectifera; G. Orchis papilionacea L.; H. Orchis ustulata L.; I. Spiranthes spiralis (L.) Chevall; J. Orchis x gennarii Reichenb. fil.

Fotos/Photos: T. Martín, J. Gila y M. Pascual.

De todos ellos se aportan comentarios corológicos generales y concretos del área geográfica en cuestión, información de su comportamiento ecológico y cuantificación de las poblaciones. Se añaden asimismo diversas consideraciones de gestión sobre factores de amenaza e información relativa a su interés conservacionista, especialmente en el caso de las orquídeas consideradas más raras y amenazadas (Decreto 63/2007; Ley 4/2015).

El presente trabajo se ha planteado con el objetivo general de contribuir a aportar novedades florísticas tanto al catálogo regional, como al catálogo de las orquídeas silvestres de Segovia, el cual se ha incrementado en 9 taxones, constando actualmente de 44 taxones incluidos en 15 géneros, que suponen el 46,8 \% del total de las especies de orquídeas silvestres ibéricas (Aedo \& Herrero, 2005). 


\section{Coeloglossum viride (L.) Hartm.}

Sg, Arcones, La Dehesa, 30TVL45, 1319 m., pastizales húmedos del margen de pequeño arroyo estacional sobre sustrato silíceo, 01.06.2011, T. Martín, Fig. 1-A.

Orquídea eurosiberiana, distribuida por el N. de América, gran parte de Asia y Europa, llegando hasta la mitad septentrional de la Península Ibérica -Pirineos, cordillera cantábrica y Sistema Ibérico -(Aedo, 2005). Citada con relativa frecuencia en la vertiente castellano-leonesa de la Cordillera Cantábrica, León, Palencia y Burgos (Aedo \& Herrero, 2005; Díez Santos, 2011; Ruíz de Gopegui et al. 2017; Anthos, 2018) y el Sistema Ibérico burgalés y soriano (Segura et al. 2000; Alejandre et al. 2006; Anthos, 2018). Más recientemente ha sido citada de la vertiente sur del Sistema Central (Sierra de Ayllón y Sierra del Rincón) en Guadalajara y Madrid (Baonza, 2012; Anthos, 2018 [Pliego-D. Galán-2010]). La localidad aportada supone la primera observación de esta orquídea en Segovia y en la Sierra de Guadarrama, así como la primera referencia para la vertiente norte del Sistema Central y la tercera para el conjunto del Sistema Central. Así mismo, constituye hasta ahora la localidad más meridional de la Península Ibérica. No obstante, existe una antigua cita (VVAA, 2018) que corresponde a la referencia bibliográfica del trabajo «Prodomus Florae Hispanicae, Vol. I» (Willkomm \& Lange, 1861-1862), en el cual a su vez se recabó la información de Quer (1784:22), que en su obra «De la Flora española o historia de las plantas de España», refiriéndose a esta orquídea con el sinónimo "Satyrium viride", describe la observación textualmente "la he visto en el circuito del Real Sitio de San Ildefonso, cerca de la fuente que llaman del Príncipe". Hemos visitado este emplazamiento en fechas recientes comprobando que todo el entorno de la Fuente del Príncipe se encuentra urbanizado, circunstancia que hace imposible su presencia y no nos permite ni siquiera poder valorar si los hábitats preexistentes eran idóneos para albergar poblaciones de esta orquídea. En Flora Iberica (Aedo \& Herrero, 2005) no se indica su presencia en la provincia de Segovia, mientras que se contempla como registro dudoso e impreciso, con buen criterio, no aceptado e incluido en el listado de citas no confirmadas (Anthos, 2018).

La población citada consta de 22 ejemplares (12 florecidos y 10 vegetativos), bastante agrupados en un solo núcleo que ocupa un espacio muy reducido de pastizal de unos $5 \mathrm{~m}^{2}$. Estos pastizales, húmedos por la influencia de un pequeño arroyo, se encuentran formando parte del estrato herbáceo de un melojar (Quercus pyrenaica Lam.) viejo y adehesado, asentado sobre sustrato silíceo, con un relieve de suave pendiente y exposición norte, que se corresponde con la comunidad vegetal de melojares supramediterráneos silicícolas (melojares guadarrámicos). Durante la observación, se pudo comprobar la existencia de herbivorismo-predación parcial o total de las inflorescencias de algunos ejemplares-, debido a la excesiva carga ganadera que soportan estas comunidades. 
Taxón que reviste especial interés biogeográfico por su carácter finícola y porque se trata de una única población con muy escaso número de efectivos. Aunque no se encuentra incluida en los listados de flora protegida, debido a las numerosas poblaciones existentes en las áreas montañosas del sector norte y este de la comunidad castellano-leonesa, este tipo de poblaciones localizadas en el límite de su área de distribución deberían tener un tratamiento de gestión y conservación diferenciado, que bien podría articularse a través de los instrumentos de gestión del espacio natural protegido Parque Natural Sierra Norte de Guadarrama.

\section{Epipactis helleborine (L.) Crantz subsp. helleborine}

SG, Riofrío de Riaza, Arroyo de la Tejera, 30TVL6466, 6566, 1430-1523 m., rebollar denso, húmedo y umbroso en sustrato silíceo, 30.06.2013, J. Gila \& T. Martín, Fig. 1-B; Cerezo de Arriba, Ermita de San Benito, 30TVL6163, 1405 m., nemoral en pequeño abedular con agrupaciones de sauces, 06.07.2015, T. Martín.

Taxón eurosiberiano ampliamente distribuido en Eurasia, que en la Península Ibérica se restringe al tercio septentrional, siendo escasa y muy localizada en C. y S. (Crespo, 2005). Citada en León (Aedo \& Herrero, 2005; Díez Santos, 2011), Palencia (Ruiz de Gopegui et al. 2017), Valladolid (Santos et al. 2008), Burgos (Alejandre et al. 2006), Soria (Segura et al. 2000; Molina et al. 2014) y Madrid (Baonza, 2007). Orquídea de compleja determinación por su amplia variabilidad morfológica y fenotípica de la que existen varias referencias bibliográficas (Cebolla, 1985; Romero \& Rico, 1989; Soriano $\&$ al., 2002) que mencionan su presencia en Segovia, más concretamente en ambientes riparios de los tramos medios de los ríos Duratón y Cega. En ambos casos, se ha comprobado una determinación errónea, debiendo atribuirse dichas referencias a Epipactis rhodanensis Gévaudan \& Robatsch, e interpreto que con idéntico criterio de revisión y asignación de citas no se ha incluido en Flora Iberica (Aedo \& Herrero, 2005). Así pues, se confirman las primeras citas de esta interesante orquídea silvestre para la provincia de Segovia. En las localidades de Riofrío de Riaza se halló en melojares densos, umbrosos y húmedos con matorral disperso de Erica arborea L. y Cytisus scoparius (L.) Link, siendo muy escasa en las dos poblaciones contabilizando 25 y 30 ejemplares respectivamente. En la localidad de Cerezo de Arriba tan sólo se observó un único ejemplar en el estrato herbáceo de un pequeño abedular.

A pesar de no encontrarse incluida en listados de flora protegida en Castilla y León, sería muy conveniente abordar un trabajo en profundidad de acreditación de la distribución real de esta orquídea, mediante la revisión, comprobación y corrección de la ingente cantidad de referencias asignadas a ella y que en realidad corresponden a otros taxones del género (E. tremolsii Pau, E. rhodanensis Gévaudan \& Robatsch, E. phyllanthes G.E. Sm. y E. kleinii M. B. Cespo), con la finalidad de precisar el conocimiento sobre la distribución y estado de sus poblaciones, evaluando en último término la necesidad de su protección. 


\section{Epipactis microphylla (Ehrh.) Swartz.}

SG, Cedillo de la Torre, Arroyo de la Hoz, 30TVL4586, 1060-1080 m., nemoral en quejigar y encinar-sabinar umbrosos, 28.06.04, T. Martín, Fig. 1-C; Santo Tomé del Puerto, El Pozuelo-río Duratón, 30TVL4762, 1000-1060 m., nemoral en encinar y encinar-quejigar con sabinas (J. thurifera) dispersas, 06.06.06, T. Martín; Santo Tomé del Puerto, Valle del río del Puerto, 30TVL4861, 1016 m., en encinar-quejigar umbroso, muy húmico, con sabinas dispersas, 21.06.09, T. Martín; Santo Tomé del Puerto, sin topónimo, 30TVL4760, 1087 m., en retazos de quejigar joven de solana, con encinas y sabinas dispersas, 22.06.10, T. Martín; Escobar de Polendos (E.L.M. Villovela de Pirón), Barranco de la Estacada, 30TVL0852, 921 m., nemoral en ambiente de encinar-sabinar, 17.07.10, T. Martín; Cedillo de la Torre, Umbría de la Hoz, 30TVL4786, 1067 m., encinar umbroso y muy húmico con sabinas dispersas, 03.06.11, T. Martín \& R. Díez; Cedillo de la Torre, Arroyo Hocecilla, 30TVL4687, 1078 m., encinar-sabinar umbroso y lecho seco de arroyo estacional, 03.06.11, R. Díez \& T. Martín; Cedillo de la Torre, Carracedillo, 30TVL4787, 1079 m., encinares arbustivos densos y umbrosos con sabinas dispersas, 03.06.11, T. Martín \& R. Díez; Montejo de la Vega de la Serrezuela, Hoces del Riaza, 30TVL5099, 890 m., en encinar calcícola con sabinas salpicadas y orla espinosa, 07.07.12, T. Martín; Montejo de la Vega de la Serrezuela, Hoces del Riaza, 30TVL5098, 882 m., encinar calcícola denso y umbroso, 07.07.12, T. Martín; Maderuelo, Barranco de Valugar, 30TVL5198, 894 m., escasa en encinar calcícola umbroso, 07.07.12, T. Martín; Arahuetes (E.L.M. Pajares de Pedraza), sin topónimo, 30TVL2857, 933 m., en encinar-sabinar calcícola denso, umbroso y muy húmico y sabinar con encinas, quejigos y fresnos, 24.06.13, T. Martín; Pedraza, sin topónimo, 30TVL3052, 1062 m., encinar viejo con poda tradicional con sabina, enebro y orla espinosa, 16.07.13, T. Martín; Pedraza, Hoya Espesa, 30TVL3053, 1080 m., muy escasa encinar-sabinar de suelo calizo profundo con sotobosque de orla espinosa, 16.07.13, T. Martín; Arahuetes (E.L.M. Pajares de Pedraza), sin topónimo, 30TVL2757, 1040 m., en encinar-sabinar calcícola, umbroso, con quejigos, 22.06.14, J. Gila; Escobar de Polendos (E.L.M. Villovela de Pirón), Barranco de la Estacada, 30TVL0752, 915 m., nemoral en ambiente de encinar-sabinar, 14.06.2015, J. Gila ; Riaza (E.L.M. El Negredo), Arroyo de Valdileja, 30TVL7474, 1153 m., en ambiente ripario de quejigar con fresnos, 15.06.15, T. Martín; Sepúlveda (E.L.M. Castrillo de Sepúlveda), El Monte, 30TVL3575, 1043 m., encinares calcícolas con suelos profundos, 08.07.15, T. Martín \& M. Pascual.

Orquídea de distribución latemediterránea, que en la Península Ibérica se encuentra en el cuadrante NE, con localidades aisladas en W y S (Aedo \& Herrero, 2005). De nuestro entorno, se tiene constancia de su presencia en León, Burgos y Soria (Segura et al. 2000; Crespo, 2005; Alejandre et al. 2006; Diez Santos, 2011; Molina et al. 2014). De Segovia, salvo tres referencias inéditas (VVAA, 2018), no se señala su presencia en Flora Ibérica (Aedo \& Herrero, 2005), ni tampoco en el listado de pliegos de 
herbario y citas bibliográficas del programa ANTHOS (Anthos, 2018). Por consiguiente, constituye novedad provincial, aportándose 18 núcleos poblacionales diferentes, referidos a cuadrículas UTM de $1 \times 1 \mathrm{Km}$., que corresponden a 8 cuadrículas UTM de $10 \times 10 \mathrm{Km}$. Estas poblaciones, además de ampliar notablemente la información sobre su distribución, representan las observaciones más meridionales de esta orquídea en territorio castellano-leonés.

Las localidades, se concentran en el cuadrante nororiental, desarrolladas sobre sustratos calcáreos desde pedregosos a profundos-ricos en materia orgánica-, en ambientes nemorales de encinares, quejigares, sabinares y todas sus variantes mixtas, del piso supramediterráneo con ombroclima subhúmedo-húmedo, en un rango altitudinal de 882-1153 m. En todas las poblaciones es característica común su escasez, habiendo contabilizado en los núcleos poblacionales donde es más abundante 40-60 ejemplares, mientras que en los núcleos con poblaciones más exiguas se hallaron 3-5 ejemplares. Incluida en los listados de flora protegida de Castilla y León en la categoría "De Atención Preferente" (Decreto 63/2007; Ley 4/2015).

\section{Epipactis tremolsii Pau}

SG., Monterrubio, Lastras de Lama, 30TUL8320, 1060 m., escasa en encinar-quejigar sobre calizas, 07.06.2002, T. Martín, Fig. 1-D; Escobar de Polendos (E.L.M. Villovela de Pirón), Monte de Villovela-Barranco de la Estacada, 30TVL0751-0752-0753-08520853-0953, 880-960 m., relativamente abundante en encinar-sabinar sobre calizas, así como en encinar-sabinar con rodales de pinos en sustrato silíceo, 08.05.03, T. Martín; Pedraza, Cercas de Valcorto-Vaillo, 30TVL3053-3054-3153, 1000-1080 m., en encinares y encinar-sabinar sobre calizas, 21-05.03, T. Martín; Sacramenia, Coto de San Bernardo, 30TVL2293-2294-2295-2393-2394-2395-2493-2494-2495, 880-948 m., nemoral en quejigares, en pequeñas manchas de encinar y en claros con matorral, en sustrato calizo, 10.06.03, T. Martín; Cuevas de Provanco, Tormazal, Carralba, Solano y Montelobaes, 30TVL2098-2099, 860 m., nemoral en quejigares sobre calizas y áreas descarnadas sujetas a procesos erosivos, 12.06.03, T. Martín; Cuevas de Provanco, Majano Alto, 30TVL1999, 850 m., en quejigar denso y zonas aclaradas sobre sustrato calizo, 12.06.03, T. Martín; Cuevas de Provanco, Cabezo, 30TVN1900-18001801, 820-880 m., quejigares sobre calizas, 12.06.03, T. Martín; Cuevas de Provanco, Valdeparada-Chorro de Extremadura, 30TVN1802-1901-1902-1903-2002-2003, 920 980 m., nemoral en quejigares arbóreos y arbustivos sobre sustrato calizo, 28.05.04, T. Martín; Otero de Herreros, Arroyo de la Escoria-arroyo del Quejigar, 30TUL96199719, 1080 m., en encinar-quejigar joven e incipiente en sustrato silíceo, 03.06.04, T. Martín; Laguna de Contreras, El Montecillo, 30TVL1295-1395-1495, 800-880 m., quejigar umbroso y quejigar-encinar con sabinas sobre calizas, 10.06.04, T. Martín; Sacramenia, Abejón, 30TVL1795-1796-1895-1896, 840-920 m., nemoral en queji- 
gares, pinares de plantación, áreas con matorral y suelos erosionados desprovistos de vegetación, 10.06.04, T. Martín; Valtiendas, La Muñeca-El Robledillo, 30TVL19901991, 820-860 m., quejigar joven y denso en ladera caliza con exposición noroeste, 23.06.04, T. Martín; Fuentidueña, El Robledillo, 30TVL1890-1990, 820-860 m., nemoral en quejigar joven en plena regeneración sobre calizas, 23.06.04, T. Martín; Valdeprados, Arroyo del Quejigal, 30TUL9520, 1000 m., quejigar-encinar con fresnos dispersos en la franja riparia, sustrato calizo, 02.07.05, T. Martín; Prádena (E.L.M. Castroserna de Arriba), El Cerezo-Arroyo Velasco, 30TVL3858-3859-3759, 1100 $1140 \mathrm{~m}$., nemoral en encinares-sabinares densos de umbría con matorral de labiadas en sustrato calizo, 22.05.06, T. Martín; Fuentesoto, El Encinar, 30TVL2389, 960 m., encinar-quejigar incipiente en calizas, 17.06.06, T. Martín; Castroserna de Abajo, sin topónimo, 30TVL3759, 1100 m., encinar-sabinar denso de umbría en sustrato calizo, 22.05.06, T. Martín; Valleruela de Sepúlveda, La Mediana, 30TVL3758-3858, 10801120 m., nemoral en encinar-quejigar de umbría, encinar-sabinar de solana y en pequeño quejigar isla, 22.05.06, T. Martín; Maderuelo-Montejo de la Vega de la Serrezuela, Valugar, 30TVL5198, 870-900 m., encinares umbrosos en calizas, 20.05.08, T. Martín; Maderuelo, Maluque, 30TVN5201-5301-5401-997-1012 m., encinar-sabinar abierto con matorral en calizas, 08.07.08, T. Martín \& R. Díez; Cedillo de la Torre, sin topónimo, 30TVL4988, 1040 m., encinar-quejigar con matorral de jaras, 19.05.11, T. Martín; Fuentidueña, Cerro San Blas, 30TVL2087, 990 m., encinar con pedrera en calizas, 17.06.12, J. Gila; Montejo de la Vega de la Serrezuela, Cerro Vallejo Sancho, 30TVL5098-5099, 880-890 m., encinar-sabinar calcícola con matorral de labiadas, 07.07.12, T. Martín; Cerezo de Arriba, sin topónimo, 30TVL5468, 1159 m., rebollar con encinas dispersas y matorral de jara y gayuba, 29.05.13, T. Martín; Riaza, sin topónimo, 30TVL5568, 1160 m., en gayubares tapizantes en claros de rebollar sobre sustrato silíceo, 11.07.13, T. Martín; Riaza (E.L.M. Alquité), La Piedra salinera, 30TVL6673-6674-6574, 1104-1181 m., nemoral en rebollar joven y denso, así como rebollar muy abierto con gayuba sobre rañas, 27.05.15, T. Martín; Ribota (E.L.M. Aldealázaro), La Dehesa, 30TVL6675-6676-6575, 1069-1101 m., rebollares sobre rañas con matorral de jara, gayuba, cantueso y tomillo, 27.05.15, T. Martín; Riaza (E.L.M. El Negredo), Arroyo de Valdileja, 30TVL7474-7574, 1162-1198 m., encinares muy abiertos, jarales de sucesión regresiva y pequeñas pedrizas silíceas, 15.06.15, T. Martín; Villacastín, Ladera de las Cárcavas, 30TUL7615-7515, 1075-1086 m., encinares supramediterráneos silicícolas de ladera con exposición de solana, 29.06.16, T. Martín; Riaza (E.L.M. Villacorta), Laderas de los Cambrones, 30TVL7074, 1118 m., rebollar degradado con jaral y áreas desprovistas de vegetación con suelos arcillosos y guijarrosos, 30.06.16, T. Martín.

Taxón cuya distribución se circunscribe al área mediterránea occidental-Península lbérica, sur de Francia, Córcega, Cerdeña y norte de África-, que se presenta disperso en buena parte de la geografía peninsular (Crespo, 2005). Citada en todas las provincias 
castellano-leonesas (Aedo \& Herrero, 2005; Bariego \& Gastón, 2005; Segura et al. 2000; Alejandre et al. 2006; Santos et al. 2008; Díez Santos, 2011; Molina et al. 2014; Ruiz de Gopegui et al. 2017), aunque respecto a la provincia de Segovia cabe significar que no se conocen referencias bibliográficas previas que mencionen su presencia. Sin embargo, aparece señalada en Flora Ibérica (Crespo, 2005) como consecuencia de la revisión realizada por Crespo en noviembre de 2003, sobre material de pliego identificado por García Adá \& G. López como Epipactis atrorubens (Bernh.) Besser con referencia MA 468340 procedente de Orejana (Orejanilla), en la que determina corresponde a Epipactis tremolsii Pau con referencia MA 24396 (Anthos, 2018). En este mismo sentido, cabe añadir que se ha comprobado que la totalidad de las citas bibliográficas (Romero \& Rico, 1989; García Adá, 1995) atribuidas a E. atrorubens, en realidad deben asignarse a $E$. tremolsii Pau. De manera que el conjunto de localidades aportadas además de constituir los primeros registros publicados totalmente constatados de esta orquídea en Segovia, contribuyen a ampliar notablemente el conocimiento sobre su distribución en la mitad norte peninsular.

Orquídea que se presenta bien distribuida en los sectores septentrional y central (69 cuadrículas UTM de 1X1 km. y 17 cuadrículas UTM de 10X10 km.), siendo muy escasa y localizada en el sector meridional. Indiferente edáfica, pero con clara preferencia por los sustratos calizos, en los que habita encinares, quejigares, encinaressabinares, formaciones mixtas de ambas quercíneas, sus claros, pedrizas y áreas de suelos desprovistas de vegetación sujetos a procesos erosivos; y en sustratos silíceos en encinares y rebollares, así como sus etapas regresivas de matorral (jarales y gayubares) y pequeñas pedrizas. Todos estos ambientes se localizan en el piedemonte serrano, cuencas medias, páramos calcáreos y sus cuestas, en el piso bioclimático supramediterráneo (800-1200 m.) bajo ombroclima seco-subhúmedo. Se encuentra incluida en los listados de flora protegida de Castilla y León en la categoría "De Atención Preferente" (Decreto 63/2007; Ley 4/2015).

\section{Ophrys fusca Link. subsp. fusca}

SG., Arahuetes, Los Castillejos, 30TVL2755, 1011 m., relativamente común en sabinar con matorral de jaras en sustrato calizo, 13.04.08, J. Gila, Fig. 1-E; Torreiglesias, Covatillas, 30TVL1049, 968 m., sabinar-encinar con quejigos dispersos, enebros y matorral de junquillo, salvia y jopillos, 24.04.09, J. Gila; Orejana, La Jalvera, 30VL3557, 1087 m., matorrales de jopillo, espliego y socarrillo, sucesión regresiva de encinar-sabinar pretérito, 09.05.09, T. Martín; Orejana, Castillejo, 30TVL3255, 1054 m., sabinar-encinar de solana con quejigos en sustrato calizo, 09.05.09, T. Martín; Torreiglesias, Covatillas, 30TVL0949, 973 m., sabinar-encinar de solana, con matorral de salvia, espliego, junquillo y jopillos en sustrato calizo, 29.05.09, J. Gila \& T. Martín; Caballar, Los Javinales, 30TVL1953, 1047 m., encinar-quejigar joven en calizas con orientación oeste, 
25.05.10, T. Martín; Pedraza, El Prado Monje, 30TVL3254, 1055 m., encinar-sabinar de solana con matorral de tomillo, salvia y jopillo, en sustrato calizo, 28.05.10, T. Martín; Rebollo, sin topónimo, 30TVL2858, 955 m., encinar-sabinar con enebro en sustrato calizo, 04.05.11, T. Martín; Cubillo, sin topónimo, 30TVL2553, 1026m., sabinar-encinar con quejigos y matorral de jara, salvia, espliego, tomillo, junquillo y jopillo, 25.05.13, J. Gila \& T. Martín; Caballar, El Valle-río del Horco, 30TVL1952, 1012 m., pastizales en claros de matorral de salvia, espliego, tomillo y jopillos, 28.04.16, T. Martín; Santiuste de Pedraza (E.L.M. Requijada), La Cantera, 30TVL2851, 1048 m., encinar-sabinar con matorral de tomillo y jopillo, en sustrato calizo, 13.05.16, T. Martín; Valleruela de Sepúlveda, Los Cotarrillos, 30TVL3559, 1123 m., fenalar con sabinas en pequeños bosquetes y dispersas y matorral de tomillos y espliego, en sustrato calizo, 30.05.18, T. Martín \& R. Díez; Sepúlveda (E.L.M. Villar de Sobrepeña), Lastrón Redondo, 30TVL3069, 1025 m., tomillares (T. zygis) con sabinas dispersas en calizas, 02.04.19, M. Pascual \& T. Martín.

Taxón distribuido por la región mediterránea occidental, se presenta disperso por casi toda la Península Ibérica, siendo raro en el NW y Baleares (Aldasoro \& Sáez, 2005). En el centro peninsular las localidades más próximas donde se ha confirmado su presencia se encuentran en las provincias de Guadalajara, Soria, Burgos y Palencia (Carrasco et al. 1997; Segura et al. 2000; Alejandre et al. 2006; Ruíz de Gopegui et al. 2017). Novedad provincial, de la que se aportan poblaciones en 13 cuadrículas UTM de 1X1 km. y 6 cuadrículas UTM de $10 \times 10$ km., contribuyendo a completar en el contexto geográfico del centro peninsular el mapa de distribución de esta orquídea en la Península Ibérica (Aldasoro \& Sáez, 2005). Todas las poblaciones halladas se encuentran muy localizadas en los afloramientos calizos-lastras, cuestas y cañones-, del sector central del piedemonte serrano, en el piso supramediterráneo (955-1055 m.) con ombroclima seco. Se presenta en poblaciones no excesivamente abundantes, cuyos individuos suelen ocupar los emplazamientos más umbrosos y frescos de formaciones mixtas de encinar-sabinar xerófilo, con quejigos y matorral compuesto por jaras, enebro, salvia, espliego, tomillos, junquillo y jopillos, y en fragmentos de las comunidades de matorral descritas, que constituyen etapas de sucesión regresiva.

\section{Ophrys insectifera L. subsp. insectifera}

SG., Ayllón (E.L.M. Grado del Pico), Sierra de Pela, 30TVL87, 1331 m., pastizales vivaces en claro de arbusteda espinosa, 05.06.09, T. Martín, Fig. 1-F.

Orquídea ampliamente distribuida por casi toda Europa, que en la Península Ibérica se encuentra presente en el tercio norte, algunos enclaves del centro-oeste y Baleares (Aldasoro \& Sáez, 2005). De nuestro entorno castellano-leonés, las referencias más próximas se sitúan en áreas montanas de la Cordillera Cantábrica y el Sistema Ibérico, en las provincias de Soria, Burgos, Palencia y León (Segura et al. 2000; Aldasoro \& 
Sáez, 2005; Alejandre et al. 2006; Díez Santos, 2011, Ruíz de Gopegui et al. 2017). Novedad provincial de relevante interés corológico y biogeográfico, que supone la ampliación del límite sur de su área de distribución en el centro peninsular y la localidad más meridional referida al ámbito geográfico de Castilla y León. La pequeña población hallada está constituida por tan sólo 7 ejemplares reproductores concentrados en un único núcleo de unos $6 \mathrm{~m}^{2}$ de superficie. La comunidad vegetal, enmarcada en el piso supramediterráneo bajo ombroclima subhúmedo, está constituida por pastizal-tomillar calcícola ecotonal en la transición de aulagar a arbusteda espinosa compuesta por Crataegus monogyna Jacq., Rosa canina L., Juniperus communis L. subsp. alpina y Rhamnus saxatilis Jacq.. Efectuadas visitas a la población en años posteriores, se pudo comprobar la existencia de diversas circunstancias meteorológicas adversas que han impedido durante varios años la normal fructificación de esta minúscula población, como una fuerte granizada a principios de junio del año 2011 que segó los tallos e inflorescencias justo en la antesis, o las persistentes olas de calor de fínales de junio de los años 2015, 2016 y 2017 que provocaron el abortado de la fructificación. Se trata por tanto de la orquídea silvestre más escasa y amenazada de Segovia, la cual se encuentra incluida en los listados de flora protegida de Castilla y León en la categoría "De Atención Preferente" (Decreto 63/2007; Ley 4/2015).

\section{Orchis papilionacea L.}

SG., Ayllón (E.L.M. Santibáñez de Ayllón), Matita, 30TVL77, 1158m., pastizales vivaces en encinares sobre sustrato silíceo, 18.05.12, T. Martín \& D. de Frutos, Fig. 1-G; Otero de Herreros, Cuesta de Valdecerra, 30TUL91, $1050 \mathrm{~m}$., pastizales pobres y pedregosos de claros de encinar silicícola, 11.05.13, T. Martín \& J. Gila; Duruelo, sin topónimo, 30TVL46, 1089 m., sabinar incipiente con encinas y quejigos en calizas, 28.05.13, D. de Frutos \& T. Martín; Arahuetes (E.L.M. Pajares de Pedraza), sin topónimo, 30TVL25, 1020 m., sabinar con denso matorral de labiadas en sustrato calizo, 05.06.13, T. Martín \& R. Sánchez; Valle de Tabladillo, Los Quemadillos, 30TVL37, 1119 m., en comunidad de pastizal-matorral con tomillos y espliego en calizas, 14.05.14, M. Pascual \& T. Martín; Navas de Riofrío, sin topónimo, 30TVL02, 1074 m., encinar con matorral de tomillar-cantuesar en sustrato silíceo, 09.05.15, T. Martín; Mata de Cuéllar, Fuente del Valle, 30TVL78, 874 m., pinar-encinar con sabinas y orla arbustiva espinosa en calizas, 29.04.16, T. Martín \& J. Rico; Santo Domingo de Pirón, Navavidrial, 30TVL14, 1131 m., berceal-cantuesar en sustrato silíceo, 09.05.16, E. Calvo \& T. Martín; Corral de Ayllón, Longanizas, 30TVL68, 992 m., pastizales vivaces pobres con encinas dispersas sobre sustrato silíceo, 13.05.16, T. Martín; Vegas de Matute, sin topónimo, 30TUL91, 1000 m., pastizales de terófitos y tomillares laxos con encinas dispersas en calizas, 18.04.17, D. de Frutos \& T. Martín; Sepúlveda (E.L.M. Villar de Sobrepeña), La Cañada, 30TVL37, 1058 m., ruderal en pastizales muy pobres sobre calizas, 12.05.17, M. Pascual \& T. Martín. 
Orquídea de distribución mediterránea, más frecuente en el sector occidental. En la Península Ibérica se presenta relativamente abundante en la mitad sur, enrareciéndose hacia el N-NE, con localidades en la mitad septentrional aisladas (Aedo, 2005). En el contexto geográfico de la submeseta norte-cuenca del Duero, es un taxón muy raro y escaso con contadas localidades en Soria, Palencia y León (Díez Santos, 2011; Molina Martín et al. 2014; Ruíz de Gopegui et al. 2017). En Segovia, existe una cita previa del año 2003 de un solo ejemplar en Honrubia de la Cuesta, buscado en años posteriores sin éxito (Benito Ayuso, 2009-2010), si bien se interpreta que dicha cita al no poder ser confirmada posteriormente no pudo quedar acreditada su presencia en la provincia. En consecuencia, las citas aportadas son las primeras referencias totalmente contrastadas de esta orquídea en Segovia, que constituyen una notable contribución biogeográfica para la comunidad castellano-leonesa, con la adición de casi tantas localidades como referencias previas. El conjunto de localidades presentan una distribución puntual y aislada de norte a sur a lo largo del piedemonte serrano y en los páramos del tercio norte provincial. Estas poblaciones, al igual que las de la mitad norte peninsular, muestran una distribución muy dispersa y están constituidas por un número muy reducido de ejemplares (1, 2, 3, 5, 6 ó 7), aunque la característica común a todas ellas cuando están compuestas por más de un ejemplar es que la estructura del núcleo poblacional es amacollada, es decir todos los individuos muy juntos y apretados, evidencia de su reproducción vegetativa (división de pseudobulbos) y la ausencia de reclutamiento mediante germinación de semillas. Se encuentra en pastizales vivaces y terofíticos más bien pobres y/o pedregosos, matorrales, encinares, sabinares y pinares antrópicos en ambiente de encinar-sabinar, tanto en sustratos calizos como silíceos, en la franja altitudinal de 874-1158 m. del piso supramediterráneo con ombroclima seco y subhúmedo. Aunque se citan 11 poblaciones, el reducido número de individuos que las componen y la comprobación de la roturación agrícola de una de las poblaciones del páramo, sugieren proponer la adopción de medidas de gestión preventiva de protección frente a los diversos factores de amenaza, apoyadas en el status de protección que le otorga su inclusión en la categoría "De atención Preferente" en los listados de flora protegida de Castilla y León (Decreto 63/2007; Ley 4/2015).

\section{Orchis ustulata L.}

SG., Cuéllar, sin topónimo, 30TUL9189, 891 m., pinares mixtos de pino albar y negral con sabinas dispersas y matorrales subseriales -salviares con junquillo-, en sustrato calizo, 17.05.13, J. Gila, R. Muñoz, C. Olmos \& T. Martín, Fig. 1-H; Cuéllar, Las Veguillas, 30TUL9089, 887 m., tomillares con salvia y pinos albares dispersos, 25.05.13, T. Martín \& J. Gila; Cuéllar, Corteza, 30TUL9190, 888 m., pinares albares con matorral de salvia en calizas, 27.05.13, T. Martín. 
Orquídea eurosiberiana, cuya distribución se circunscribe a la mitad norte peninsular con localidades aisladas en la Comunidad Valenciana y Andalucía (Aedo, 2005). En Castilla y León está presente en todas las provincias, más abundante en el tercio norte, siendo escasa y muy localizada en el centro y sur (Segura et al. 2000; Aedo, 2005; Bariego \& Gastón, 2005; Alejandre et al. 2006; Santos et al. 2008; Díez Santos 2011; Ruíz de Gopegui et al. 2017). Novedad provincial, de la que se aportan 3 localidades contiguas localizadas en el extremo noroccidental, que contribuyen a completar el mapa de su distribución en la comunidad castellano-leonesa. Habita en páramos calizos en ambiente de pinares albares ( $P$. pinea L.), pinares mixtos ( $P$. pinea L. y $P$. pinaster Aiton) y comunidades de matorral denso -salviares y tomillares-, en el piso supramediterráneo $(890 \mathrm{~m}$.) bajo ombroclima seco. Se trata de una orquídea muy escasa y localizada en Segovia, cuyo censo arrojó unas cifras muy pobres de 3, 4 y 130 individuos respectivamente. Aún reconociendo que a escala regional no es una orquídea amenazada, su presencia puntal y aislada en los sectores central y sur de la comunidad, con núcleos muy distantes y constituidos por un número reducido de individuos, debieran ser argumentos suficientes para considerar conveniente un tratamiento de protección y gestión diferenciado dirigido a evitar extinciones locales.

\section{Spiranthes spiralis (L.) Chevall}

SG., Riaza, Ermita de Hontanares, 30TVL6370, 1420 m., pastizales y jarales en claros de rebollar, 07.09.06, T. Martín, Fig. 1-I; Riaza, Monte de la Comunidad, 30TVL5763, 1260 m., rebollar claro con matorral de brecina y enebro rastrero, 07.09.06, T. Martín; Carabias (E.L.M. Pradales), El Juncal, 30TVL4289, 1160 m., pastizales vivaces en ambiente ecotonal de rebollar, 07.09.06, T. Martín; Torreadrada, La Dehesa, 30TVL3288, 1120 m., pastizales y jarales en ambiente de rebollar, 20.06.06, T. Martín; Ortigosa del Monte, El Berrocal, 30TUL9922, 1046 m., muy escasa en ambiente de encinar-quejigar, 11.11.07, T. Martín; San Ildefonso, Robledo, 30TVL1329, 1119 m., muy escasa en pastizal vivaz en claro de rebollar, 25.09.10, T. Martín; Aldeanueva de la Serrezuela, La Veguilla, 30TVL3690, 1154 m., pastizales húmedos en claros de arbustedas espinosas, 08.10.10, T. Martín; Castillejo de Mesleón, sin topónimo, 30TVL5070, 1059 m., pastizales en claros de rebollar-encinar muy abierto, 16.09.14, T. Martín \& R. Díez; Cerezo de Arriba, La Gamonosa, 30TVL5367, 1069 m., en pastizales vivaces bajo rebollar, 16.09.14, T. Martín \& R. Díez; Ayllón, Río Vadillo, 30TVL7075, 1066 m., pastizales vivaces en fresneda riparia, 03.10.14, T. Martín \& R. Díez; Carabias (E.L.M. Pradales), Arroyo de la Fuente del Risco, 30TVL4189-4190, 1142 m., abundante en comunidades de praderas-juncales, 13.10.14, T. Martín \& R. Díez; Carabias (E.L.M. Pradales), sin topónimo, 30TVL3990, 1160 m., ruderal en pastizales de borde de camino, 13.10.14, T. Martín \& R. Díez; Aldeanueva de la Serrezuela, sin topónimo, 30TVL3492, 1063 m., relativamente abundante en claros de 
encinar-rebollar, 13.10.14, T. Martín \& R. Díez; Aldehorno, Fuentelpie, 30TV3494, 1025 m., pastizales ralos en claros de encinar-rebollar, 13.10.14, T. Martín \& R. Díez; Grajera, Cerro Valderríes, 30TVL5179, 1020 m., en claros de rebollar con matorral de tomillo y cantueso, 13.10.14, T. Martín \& R. Díez; Grajera, Cerro de Terradillos, 30TVL5080, 1025 m., pastizales de diente en claros de rebollar muy abierto, 23.10.14, T. Martín; Santo Tomé del Puerto, Las Cercanas, 30TVL5058, 1154 m., pastizales vivaces con encinas dispersas y orla espinosa, 23.10.14, T. Martín; Corral de Ayllón, sin topónimo, 30TVL6383, 993 m., pastizales vivaces con encinas dispersas, 02.11.14, T. Martín; Riaza, La Poba, 30TVL6066, 1270 m., claros de rebollar denso con matorral de rascaviejas, 03.11.14, T. Martín; Prádena, El Chorro, 30TVL4153, 1089 m., sabinar con encinas dispersas en sustrato calizo, 18.09.15, T. Martín; Ayllón (E.L.M. Grado del Pico), Los Terneros, 30TVL8072, 1343 m., pastizales vivaces y matorral de jaras en calizas, 04.11.16, T. Martín; Ayllón, Divisa, 30TVL7289, 1083 m., pastizales vivaces con matorral de tomillo y cantueso, 18.09.15, T. Martín; Navares de Enmedio, Valdeslasfuentes, 30TVL3788, 1205 m., pastizales bajo rodal de rebollo, 12.09.17, T. Martín; Navares de las Cuevas, Arroyo de las Hoyadas, 30TVL3787, 1188 m., pastizales vivaces y rebollar abierto, 12.09.17, T. Martín; Navares de las Cuevas, Arroyo de Matasancho, 30TVL3786, 1158 m., pastizales en claros de rebollar, 12.09.17, T. Martín; Navares de las Cuevas, sin topónimo, 30TVL3684, 1129 m., pastizales terofíticos de claros de rebollar, 12.09.17, T. Martín; Urueñas, Vega Rambela, 30TVL3577, 1085 m., pastizales vivaces densos en sustrato calizo, 11.10.17, M. Pascual \& T. Martín; Navares de las Cuevas, Malamargo, 30TVL3683, 1124 m., pastizales en claros de rebollar, 11.10.17, T. Martín \& M. Pascual; Castroserracín, Cruz de la Calzada, 30TVL3283, 1165 m., pastizales con matorral de brecina, cantueso y jaguarzo, 11.10.17, T. Martín \& M. Pascual; Corral de Ayllón, Los Llanos, 30TVL6285, 1001 m., pastizales laxos con encinas dispersas, 24.01.19, T. Martín.

Orquídea mediterránea-atlántica bien distribuida por casi toda la Península Ibérica, más frecuente en el N, W y Baleares (Patallo \& Aedo, 2005). De territorio castellanoleonés se conoce su presencia en Ávila, Burgos, León, Palencia, Salamanca, Soria y Zamora (Segura et al. 2000; Patallo \& Aedo, 2005; Bariego \& Gastón, 2005; Alejandre et al. 2006; Díez Santos, 2011; Ruíz de Gopegui et al. 2017). Respecto a Segovia, se indica su presencia en Flora Iberica (Patallo \& Aedo, 2005), pero no se conocen citas o referencias bibliográficas, ni tampoco pliegos de herbario (Anthos, 2018) que atestigüen su presencia en la provincia. Por ello, el conjunto de localidades aportadas (31 cuadrículas UTM de 1X1 Km.; 18 cuadrículas UTM de 10X10 Km.) han de considerarse como primeras citas provinciales confirmadas. Hasta ahora, presenta una distribución restringida al cuadrante noreste provincial, donde se conocen numerosas poblaciones llegando a ser localmente abundante, a excepción de un par de localidades aisladas en el tercio sur que albergan escasos efectivos (1-5 individuos). Se presenta en un rango altitudinal de 993-1420 m. correspondiente al piso supramediterráneo, bajo 
ombroclima seco y subhúmedo. Ha sido hallada en diversidad de ambientes, pastizales vivaces y anuales, tanto secos como húmedos e incluso ruderales, praderas-juncales, comunidades mixtas de pastizal-matorral (jarales, tomillares, cantuesares y biercolares), rebollares y sus ambientes ecotonales, formaciones mixtas de encinar-rebollar y encinar-quejigar, fresnedas riparias y sabinares. Indiferente edáfico, aunque en el contexto provincial muestra una clara preferencia por los sustratos silíceos.

Orquídea no amenazada, de difícil detectabilidad debido a su fenología floral tardo estival-otoñal y a su aspecto críptico (pequeño porte, coloración y pelosidad), de la que a buen seguro se irán aportando nuevas localidades en los próximos años.

\section{Orchis x gennarii Reichenb. fil. (Orchis morio x O. papilionacea)}

SG., Valle de Tabladillo, Los Quemadillos, 30TVL37, 1119 m., 2 ejemplares en pastizales con matorral de tomillos (T. zygis, T. mastichina) en sustrato calizo, 14.05.14, M. Pascual, T. Martín \& R. Díez, Fig. 1-J; Otero de Herreros, sin topónimo, 30TUL91, 1100 m., 6 ejemplares en pastizales con encinas dispersas en sustrato silíceo, 13.05.16, T. Martín \& H. Iglesias; Sepúlveda (E.L.M. Villar de Sobrepeña), La Cañada, 30TVL37, 1058 m., 7 ejemplares en pastizales pedregosos calizos, 12.05.17, M. Pascual \& T. Martín; Arahuetes (E.L.M. Pajares de Pedraza), sin topónimo, 30TVL25, 1020 m., sabinar con denso matorral de labiadas en sustrato calizo, 11.05.19, T. Martín \& R. Sánchez.

Taxón híbrido nuevo para la provincia de Segovia, del cual en el territorio castellanoleonés únicamente se conoce que haya sido mencionada su presencia en León (Díez Santos, 2011). Su distribución provincial hasta ahora conocida se limita al páramo calizo de la cuenca media del río Duratón y al piedemonte del sector sur de la sierra de Guadarrama, aunque la coincidencia de sus progenitores en otros emplazamientos hace previsible la localización de nuevas poblaciones en cualquier momento. Se presenta en comunidades de pastizales y herbazales vivaces ligados a tomillares y en claros de encinares, entre sus parentales o en áreas próximas. Las poblaciones albergan un número muy reducido de ejemplares $(2,4,6$ y 7$)$, de aspecto variable pero con características intermedias entre ambos taxones, relativas al tamaño de plantas y flores, coloración y sobretodo la forma y tamaño del labelo. 


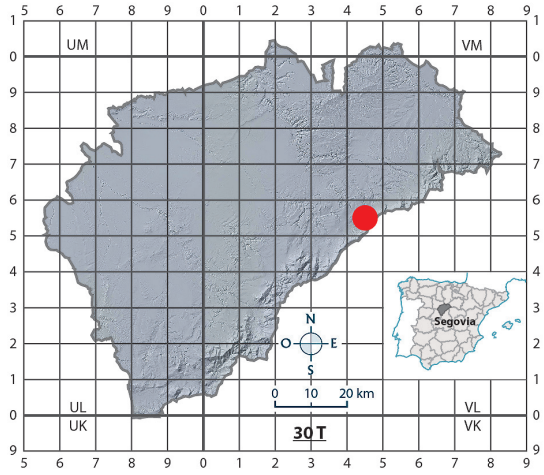

Coeloglossum viride (L.) Hartm.

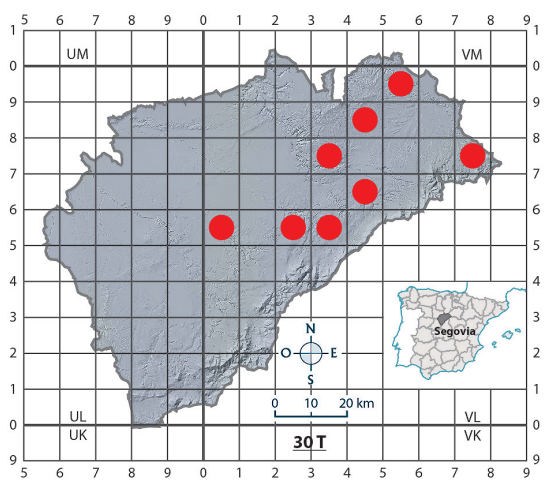

Epipactis microphylla (Ehrh.) Swartz.

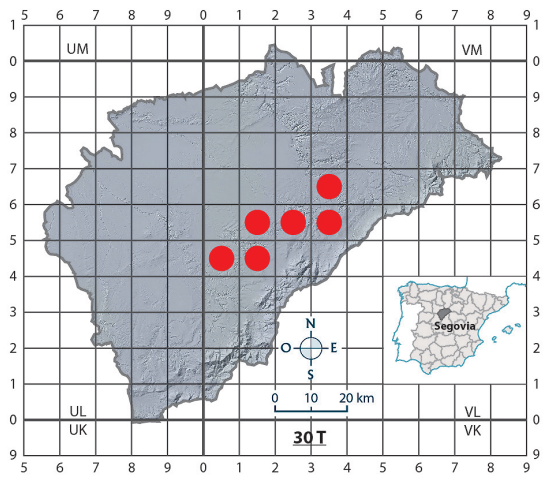

Ophrys fusca Link. subsp. fusca

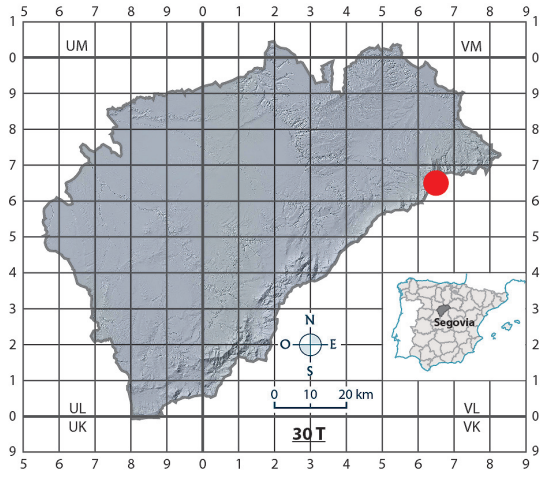

Epipactis helleborine (L.) Crantz subsp. helleborin

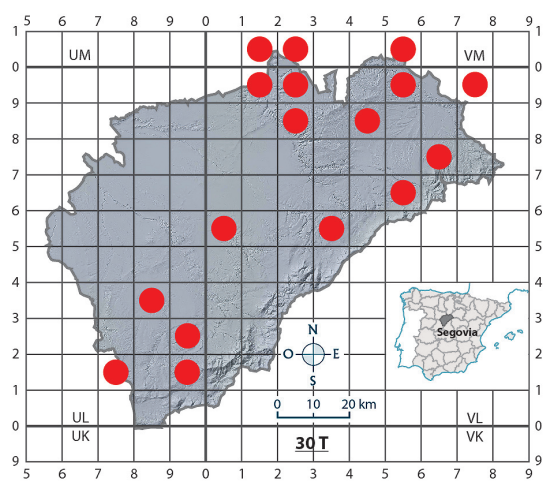

Epipactis tremolsii $\mathrm{Pau}$

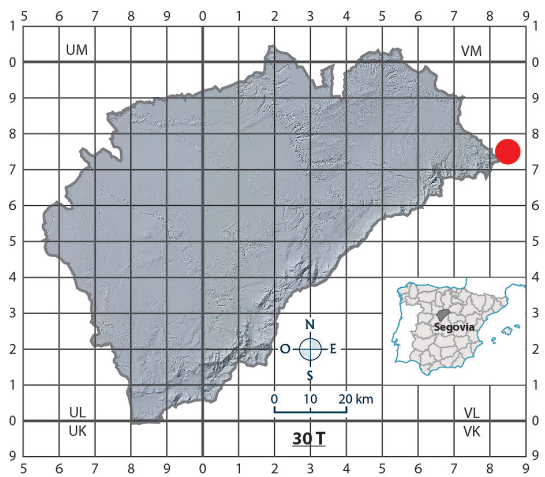

Ophrys insectifera L. subsp. insectifera

Fig. 2.1.- Mapas de distribución en cuadrículas UTM de $10 \times 10 \mathrm{~km}$., de las orquídeas silvestres citadas en la provincia de Segovia.

Fig. 2.1.- Distribution maps in 10x10 Km UTM grid cells of wild orchids recorded in the province of Segovia. 


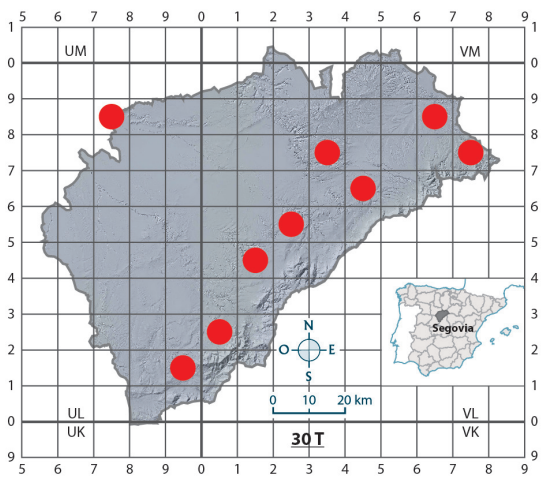

Orchis papilionacea $\mathrm{L}$.

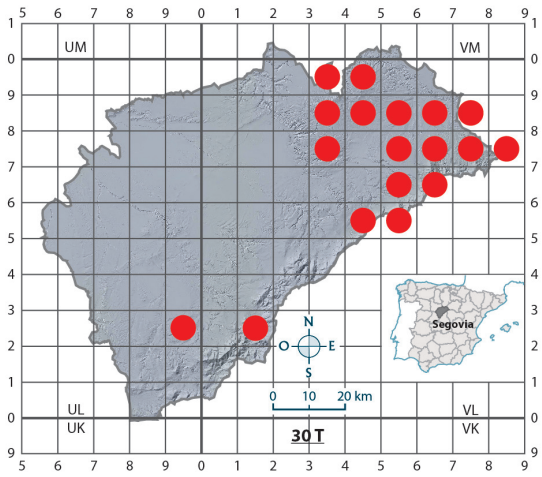

Spiranthes spiralis (L.) Chevall.

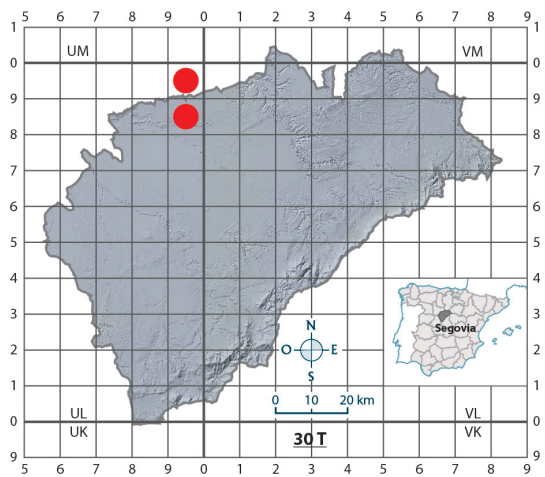

Orchis ustulata L.

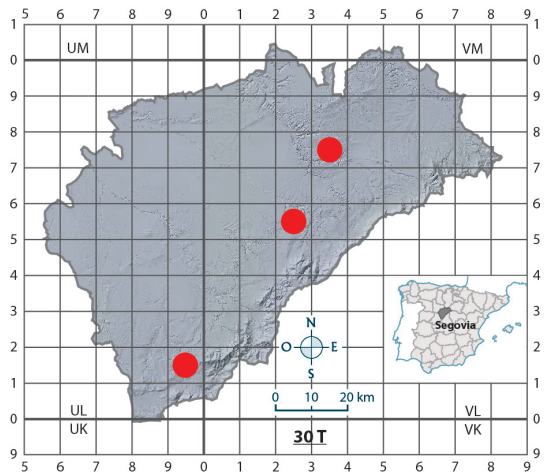

Orchis $x$ gennarii Reichenb. Fil.

Fig. 2.2.- Mapas de distribución en cuadrículas UTM de $10 \times 10 \mathrm{~km}$., de las orquídeas silvestres citadas en la provincia de Segovia (continuación).

Fig. 2.2.- Distribution maps in 10×10 Km UTM grid cells of wild orchids recorded in the province of Segovia (continued).

\section{Agradecimientos}

Nuestro agradecimiento a David de Frutos Escobar, Javier Rico Barrueta y Rafael Sánchez Payo (Agentes Medioambientales), Jesús Lorenzo Gómez y Enrique Calvo Bleye (Celadores de Medio Ambiente) y Miguel Pascual Sacristán por facilitarnos información precisa y detallada de algunas localidades. A Rafael Díez Dominguez, Honorio Iglesias Arribas, Miguel Pascual Sacristán, Carlos Olmos Otero y Raúl Muñoz Olmos por sus valiosas observaciones en numerosas excursiones botánicas. A Javier Cubo por su inestimable ayuda en la elaboración detallada de los mapas. Y a Emilio Blanco Castro y Helios Saínz Ollero por sus comentarios y la revisión crítica de los textos. 


\section{Bibliografía}

Aedo, C., Herrero, A. (Eds.). 2005. Flora Iberica Vol. XXI. Real Jardín Botánico de Madrid, CSIC. Madrid.

Aedo, C. 2005. Coeloglossum. En: Flora Iberica 21. Aedo, C., Herrero, A. (Eds.): 92-94.

Aedo, C. 2005. Orchis. En: Flora Iberica 21. Aedo, C., Herrero, A. (Eds.): 114-146.

Aldasoro, J.J., Sáez, L. 2005. Ophrys. En: Aedo, C., Herrero, A. (Eds.). Flora Iberica 21:165195.

Alejandre Sáenz, J.A., García-López, J.M., Mateo Sanz, G. (Ed.). 2006. Atlas de la flora vascular silvestre de Burgos. Junta de Castilla y León. Caja Rural Burgos. Burgos.

Anthos. 2018. Sistema de información de las plantas de España. Real Jardín Botánico-CSICFundación Biodiversidad. Recurso electrónico en www.anthos.es, consulta realizada en [29-01-2018].

Baonza, J. 2007. Algunas plantas raras en la Comunidad de Madrid. Nuevas citas y revisión corológica. Botánica Complutensis 31:87-95.

Baonza, J. 2012. Citas de flora vascular de interés de la Sierra del Rincón (Madrid, España). Botánica Complutensis 36: 105-111.

Bariego, P., Gastón, A. 2005. Aproximación al catálogo de las orquídeas silvestres de la provincia de Zamora. Ecología 19:149-164.

Benito Ayuso, J. 2009-2010. Apuntes sobre orquídeas ibéricas III. Est. Mus. Cienc. Nat. Álava 23: 49-59.

BOLETíN OFICIAL DE CASTILLA Y LEON. Decreto 63/2007, de 14 de junio, por el que se crean el Catálogo de Flora Protegida de Castilla y León y la figura de protección denominada Microrreserva de Flora.

BOLETíN OFICIAL DE CASTILLA Y LEON. Ley 4/2015, de 24 de marzo, del Patrimonio Natural de Castilla y León.

Carrasco, M.A., Macía, M.J., Velayos, M. 1997. Listado de las plantas vasculares de Guadalajara. Monografías de Flora Montiberica. Valencia.

Cebolla, C., Rivas, M.A., Soriano, C. 1984. Catálogo florístico del cañón del río Duratón (Segovia). Alismataceae a Orchidaceae. Lazaroa 6: 205-215.

Crespo, M. B. 2005. Epipactis. En: Aedo, C., Herrero, A. (Eds.). Flora Iberica 21:22-54.

Díez Santos, J.M. 2011. Guía de las Orquídeas Silvestres de la Montaña Oriental Leonesa. Grupo de Acción Local Montaña de Riaño. León.

Gabriel y Galán, J.M., Puelles, M. 1996. Catálogo florístico de las hoces del río Riaza y su entorno (Segovia). Ecología 10:273-300. 
García Adá, R. 1995. Estudio de la flora y la vegetación de las cuencas alta y media de los ríos Eresma, Pirón y Cega (Segovia). Tesis Doctoral. Universidad Complutense de Madrid. Madrid.

Molina, C., Benito, J., Montamarta, G., 2014. Aportación al catálogo de las orquídeas silvestres de la provincia de Soria. Flora Montiberica 56:91-105.

Patallo, J., Aedo, C. 2005. Spiranthes. En: Aedo, C., Herrero, A. (Eds.). Flora Iberica 21:6972.

Quer, J. 1784. De la flora española o historia de las plantas de España. Tomo VI: 22. Madrid. Romero Martín, T., Rico Hernández, E. 1989. Flora de la Cuenca del río Duratón. Ruizia 8. Monografías del Real Jardín Botánico de Madrid, Madrid.

Ruíz de Gopegui, J.A., Rodriguez, A., Alvarez, E., Velez, M., Bariego, P. 2017. Aproximación al catálogo de las orquídeas silvestres de la provincia de Palencia (Castilla y León, España). Flora Montiberica 66: 11-46.

Santos, M., Bariego, P., Delgado, L., Gavilán, L.P., Gastón, A. 2008. Aproximación al catálogo de las orquídeas de la provincia de Valladolid. Botánica Complutensis 32: 139-147.

Segura, A., Mateo, G., Benito, J.L. 2000. Catálogo florístico de la provincia de Soria (2 ed.). Excma. Diputación Provincial de Soria. Soria.

Soriano, C., Gastón, A., Bariego, P., Herrero, B., García-Viñas, J.I. 2002. Catálogo florístico del barranco del río Cega (Segovia, España). Ecología 16: 153-220.

VAA-2018. Bases de Datos de Flora Vascular Silvestre y Cartografía Detallada de Hábitats de Castilla y León. Junta de Castilla y León. 2001-2013.

Willkomm, M., Lange, J. 1861-1862. Prodomus Florae Hispanicae I: 171. Stuttgart.

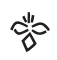

Fecha de recepcion/Date of reception: 11/03/2019

Fecha de aceptacion/Date of acceptance: 18/10/2019

Editor Asociado/Associate editor: Cristina Herrero-Jáuregui 